\title{
A CHARACTERIZATION OF UNISERIAL RINGS VIA CONTINUOUS AND DISCRETE MODULES
}

\author{
S. K. JAIN, S. R. LOPEZ-PERMOUTH and S. TARIQ RIZVI
}

(Received 22 March 1989; revised 24 August 1989)

Communicated by B. J. Gardner

\begin{abstract}
It is well-known that quasi-Frobenius rings are characterized by the property that all propective right modules are injective, as well as by the property that all injective right modules are projective. Similarly, either the property that every quasi-projective is quasi-injective or that every quasi-injective is quasi-projective characterizes uniserial rings. Oshiro has given similar characterizations for generalized uniserial rings. The purpose of this paper is to characterize rings for which continuous right modules are discrete. We show that these rings are precisely the uniserial rings. The property that every discrete module is continuous is also investigated.
\end{abstract}

1980 Mathematics subject classification (Amer. Math. Soc.) (1985 Revision): 16 A 35, 16 A 36, 16 A 50,16 A 51,16 A 52.

\section{Introduction}

The purpose of this paper is to characterize rings for which continuous right modules are discrete (that is, dual-continuous). We show that these rings are precisely the uniserial rings (Theorem 3.3). For a ring $R$ the property that every discrete right $R$-module is continuous is also equivalent to $R$ being uniserial when either $R$ is right perfect or $R$ is semiperfect with finitely generated radical (Theorem 3.8). A comprehensive treatment of discrete and continuous modules may be found in [15].

(C) 1991 Australian Mathematical Society $0263-6115 / 91 \$ A 2.00+0.00$ 


\section{Definitions and preliminaries}

Throughout this paper all rings have 1 and modules are right unital unless otherwise stated. The Jacobson radical of a ring $R$ is denoted by $J(R)$ or simply by $J$. For any $R$-module $M, \operatorname{Rad}(M), \operatorname{Soc}(M)$, and $E(M)$ denote its radical, socle, and injective hull respectively. A module $M$ is said to be valuation (also called uniserial) if its submodules are linearly ordered under inclusion. A ring $R$ is said to be right serial if it is a direct sum of valuation right $R$-modules and serial if it is both left and right serial. An artinian ring in which each one-sided ideal is principal is called a uniserial ring. It is well-known that a uniserial ring is serial. Artinian serial rings are called generalized uniserial.

For $R$-modules $N$ and $M$, we write $N \subset^{\prime} M\left(N \subset^{\oplus} M\right)$ to denote that $N$ is essential in $M(N$ is a summand of $M)$. For a right module $M$, consider the following conditions:

$\left(\mathrm{C}_{1}\right)$ For every submodule $N$ of $M$ there exists $M_{1} \subset^{\oplus} M$ with $N \subset^{\prime} M_{1}$.

$\left(\mathrm{C}_{2}\right)$ For any summand $M^{\prime}$ of $M$, every exact sequence $0 \rightarrow M^{\prime} \rightarrow M$ splits.

$\left(\mathrm{C}_{3}\right)$ If $M_{1}$ and $M_{2}$ are summands of $M$ with $M_{1} \cap M_{2}=0$, then $M_{1}+M_{2}$ is a summand of $M$.

A module $M$ is called continuous (quasi-continuous) if it satisfies $\left(\mathrm{C}_{1}\right)$ and $\left(\mathrm{C}_{2}\right)\left(\left(\mathrm{C}_{1}\right)\right.$ and $\left.\left(\mathrm{C}_{3}\right)\right)$. These conditions are dualized as follows.

$\left(\mathrm{D}_{1}\right)$ For every submodule $N$ of $M$, there exists a decomposition $M=$ $M_{1} \oplus M_{2}$ such that $M_{1} \subset N$ and $M_{2} \cap N$ is small in $M_{2}$.

$\left(\mathrm{D}_{2}\right)$ For any summand $M^{\prime}$ of $M$, every exact sequence $M \rightarrow M^{\prime} \rightarrow 0$ splits.

$\left(\mathrm{D}_{3}\right)$ If $M_{1}$ and $M_{2}$ are summands of $M$ with $M_{1}+M_{2}=M$, then $M_{1} \cap M_{2}$ is a summand of $M$.

A module $M$ is called discrete (quasi-discrete) if $M$ satisfies $\left(\mathrm{D}_{1}\right)$ and $\left(\mathrm{D}_{2}\right)$ $\left(\left(D_{1}\right)\right.$ and $\left.\left(D_{3}\right)\right)$. Discrete (quasi-discrete) modules, have also been called dual continuous (respectively quasi-dual continuous) ([13], [14]). It is easy to see that $\left(C_{2}\right)$ implies $\left(C_{3}\right)$ and $\left(D_{2}\right)$ implies $\left(D_{3}\right)$. Therefore, the following hierarchy exists:

Injective $\Rightarrow$ quasi-injective $\Rightarrow$ continuous $\Rightarrow$ quasi-continuous,

and

Projective $\Rightarrow$ quasi-projective $\nRightarrow$ discrete $\Rightarrow$ quasi-discrete. 
Notice that the condition $\left(\mathrm{D}_{1}\right)$ is equivalent to

$\left(\mathrm{D}_{1}^{\prime}\right)$ every submodule $N$ of $M$ can be written as $N=N_{1}+N_{2}$ where $N_{1} \subset^{\oplus} M$, and $N_{2}$ is small in $M$.

We list below some well-known results which will be used frequently.

2.1. Proposition [13, Propositions 1.2 and 1.5]. If $M=\bigoplus \sum M_{i}$ is (quasi-) discrete then $M_{i}$ is (quasi-) discrete and $M_{j}$-projective for $i \neq j$.

2.2. Proposition [8, Proposition 1.7 and 1.12]. If $M=\bigoplus \sum M_{i}$ is (quasi-) continuous, then each $M_{i}$ is (quasi-) continuous and $M_{j}$-injective for $i \neq j$.

2.3. Proposition [7, Proposition 3.1]. If $M$ is $M_{i}$-projective for all $i \in I$ and $M$ is finitely generated then $M$ is $\bigoplus \sum M_{i}$-projective.

2.4. Proposition [13, Theorem 2.2]. Every quasi-discrete module $M$ has a decomposition $M=\bigoplus \sum M_{i}$, where each $M_{i}$ is indecomposable. Further, this decomposition complements summands.

2.5. Proposition [14, Theorem 2.3]. A ring $R$ is right (semi-) perfect if and only if every (finitely generated) quasi-projective $R$-module is discrete.

2.6. Proposition ([5, Theorem 5.1] and [1, Proposition 2.6]). A ring $R$ is (generalized) uniserial if and only if every (indecomposable) quasi-projective right module is quasi-injective, if and only if every (indecomposable) quasiinjective right module is quasi-projective.

2.7. Proposition [4, Theorem 25.4.2]. If $R$ is a generalized uniserial ring then each $R$-module is a direct sum of cyclic valuation modules.

\section{Main results}

We start by stating two of our main theorems.

3.1. THEOREM. For a ring $R$, the following are equivalent:

(1) $R$ is uniserial;

(2) every quasi-injective module is quasi-discrete;

(3) every quasi-injective module is discrete;

(4) every continuous module is quasi-discrete;

(5) every continuous module is discrete; 
(6) every quasi-continuous module is quasi-discrete;

(7) every quasi-continuous module is discrete.

3.2. THEOREM. Let $R$ be a semiperfect ring such that $J=J(R)$ is finitely generated. Then the following conditions are equivalent:

(1) $R$ is a uniserial ring,

(2) every (quasi-) discrete $R$-module is quasi-injective;

(3) every (quasi-) discrete $R$-module is (quasi)-continuous;

(4) every finitely generated quasi-projective $R$-module is (quasi)-continuous;

(5) every quasi-projective $R$-module is (quasi-) continuous.

Before we prove these theorems we prove a lemma.

\subsection{LemMA. If $R$ is a generalized uniserial ring then}

(i) every quasi-discrete $R$-module is quasi-projective, and

(ii) every quasi-continuous $R$-module is quasi-injective.

Proof. Let $M$ be an $R$-module. Since $R$ is a generalized uniserial ring, we write $M=\bigoplus \sum_{i} M_{i}$, where each $M_{i}$ is a cyclic valuation submodule.

(i) Assume $M$ is quasi-discrete. Then by Proposition 2.1, $M_{i}$ is $M_{j}$ projective for $i \neq j$. Also, since $M_{i}$ is indecomposable, $M_{i}$ is $M_{i}$-projective by [4, Exercise 25.0.4(b)] and [10, Theorem 1.10]. Furthermore, because $M_{i}$ is cyclic, $M_{i}$ is $M=\bigoplus \sum M_{i}$-projective. But then $M$ is also $M$-projective, that is, $M$ is quasi-projective as desired.

(ii) Assume now $M$ is quasi-continuous.

This implies $M_{i}$ is $M_{j}$-injective for $i \neq j$ (Proposition 2.2). Again as in (i), $M_{i}$ is $M_{i}$-injective. Now, because $R$ is noetherian, $E(M)=$ $\bigoplus \sum_{i} E\left(M_{i}\right)$. Let $\left(\varphi_{i j}\right) \in \operatorname{End}(E(M))$ where $\varphi_{i j} \in \operatorname{Hom}\left(E\left(M_{j}\right), E\left(M_{i}\right)\right)$, and let $\sum x_{k} \in \bigoplus \sum M_{i}$. Then $\left(\varphi_{i j}\right)\left(\sum x_{k}\right)=\sum \varphi_{i k} x_{k} \in \bigoplus \sum M_{i}$, since $M_{i}$ is $M_{k}$-injective. Hence $M=\bigoplus \sum M_{i}$ is quasi-injective.

Proof OF THEOREM 3.1. Since any one of the statements (3) through (7) implies statement (2) and statement (1) implies all others (Lemma 3.3), all we need to show is that $R$ is uniserial if each quasi-injective $R$-module is quasi-discrete.

Assuming (2), we have that $R$ must be generalized uniserial [16, Theorem 2]. It then follows from Lemma 3.3 that every quasi-injective $R$ module is quasi-projective, and hence $R$ is uniserial.

PROOF OF THEOREM 3.2.

(1) $\Rightarrow(2)$. If $M$ is (quasi-) discrete then $M$ is quasi-projective (Lemma 3.3) and therefore $M$ is quasi-injective since $R$ is uniserial.

$(2) \Rightarrow(3)$ is trivial. 
(3) $\Rightarrow(4)$. Now $M$ is finitely generated quasi-projective so $M$ is discrete (Proposition 2.5), and hence $M$ is continuous.

(4) $\Rightarrow$ (1). Since $R$ is semiperfect, we can write $R=\bigoplus \sum e_{i} R$, where $e_{i} R$ is indecomposable and $e_{i} e_{j}=\delta_{i j} e_{i}$ for $i, j=1, \ldots, n$. Now, $\left(e_{i} R\right) /\left(e_{i} J^{m}\right)$ is a finitely generated quasi-projective module. Thus by hypothesis, $\left(e_{i} R\right) /\left(e_{i} J^{m}\right)$ is quasi-continuous and hence uniform. We proceed to show that $\left(e_{i} R\right) /\left(e_{i} J^{m}\right)$ is valuation and noetherian.

Set $J^{\omega}=\bigcap_{k=1}^{\infty} J^{k}$. Let $0 \neq A / e_{i} J^{\omega} \subset e_{i} R / e_{i} J^{\omega}$. There exists a positive integer $k$ such that $A \not \subset e_{i} J^{k}$. Choose the smallest $s$ such that $A \not \subset e_{i} J^{s}$. Therefore, $A \subset e_{i} J^{s-1}$. Furthermore, $\operatorname{Rad}\left(e_{i} J^{s-1}\right)=e_{i} J^{s}$, since $R$ is semiperfect. Also, $\left(e_{i} J^{s-1}\right) /\left(e_{i} J^{s}\right)$ is a completely reducible $R / J$ - (and hence $R$-) module which must be simple since $e_{i} R / e_{i} J^{s}$ is uniform. This yields that $e_{i} J^{s}$ is the unique maximal submodule of $e_{i} J^{s-1}$ which is small since $J$ and hence $e_{i} J^{s-1}$ are finitely generated. Thus $A+e_{i} J^{s}=e_{i} J^{s-1}$ implies $A=e_{i} J^{s-1}$. Therefore, every nonzero submodule of $e_{i} R / e_{j} J^{\omega}$ is of the form $\left(e_{i} J^{s}\right) /\left(e_{i} J^{w}\right)$. This yields that $e_{i} R / e_{i} J^{\omega}$ is noetherian and valuation. Therefore, $R / J^{\omega}$ is a right noetherian right serial ring. Moreover, by hypothesis, $R / J^{\omega} \times R / J^{\omega}$ is quasi-continuous. Therefore, by Proposition $2.2, R / J^{\omega}$ is self-injective and so it is quasi-Frobenius. This implies that there exists $t>0$ such that

$$
J^{t}=J^{t+1}=\cdots \text {. }
$$

But then, by the Nakayama Lemma, $J^{t}=0$, which yields $J^{\omega}=0$. Consequently, $R$ is quasi-Frobenius. Furthermore, for any ideal $A$ of $R, R / A$ is also self-injective since $R / A \times R / A$ is quasi-continuous. This gives that $R / A$ is quasi-Frobenius. Hence, $R$ is uniserial [4, Proposition 25.4.6(B)].

Finally, $(1) \Rightarrow(5)$ follows from the fact that over uniserial rings quasiprojectives are quasi-injective, while $(5) \Rightarrow(4)$ is obvious. This completes the proof.

The following example shows that the hypothesis that $J$ is finitely generated may not be removed from Theorem 3.2.

3.4. EXAmple. Let $F$ be a field. Let $\Omega=\left\{I \subset R^{+} \cup\{0\} \mid I\right.$ is wellordered $\}$. Let $T$ be the set of formal power series $\sum a_{i} x^{i}$ over $F$, with $I \in \Omega$. It is well known that for every nonzero ideal $A$ of $T, T / A$ is selfinjective [11]. Let $R=T / x T$; then $R$ is a local, non-noetherian, commutative ring satisfying the condition that every one of its homomorphic images is self-injective. Since any finitely generated quasi-projective $R$-module $M$ is a direct sum of copies of $R / B$, where $B$ is an ideal of $R$ [10], we obtain that $M$ is quasi-injective. Therefore, $R$ is a non-uniserial ring satisfying statement (4) of Theorem 3.2. 
In the next theorem we trade the hypothesis of having a finitely generated radical for that of being perfect.

3.5. THEOREM. For a ring $R$ the following conditions are equivalent:

(1) $R$ is uniserial;

(2) every (quasi-) discrete $R$-module is quasi-injective and $R$ is right perfect;

(3) every (quasi-) discrete $R$-module is (quasi-)continuous and $R$ is right perfect;

(4) every quasi-projective $R$-module is (quasi-)continuous.

Proof. Note $(1) \Rightarrow(2)$ follows by Lemma $3.3,(2) \Rightarrow(3)$ is trivial and (3) $\Rightarrow$ (4) follows from Proposition 2.5. Thus we only need to verify (4) $\Rightarrow$ (1).

Assume (4). Let $A$ be an ideal of $R$ and $S$ a direct sum of copies of $R / A$. Since $S$ is quasi-projective, it is quasi-continuous. It follows from Proposition 2.2 that every free $R / A$ module is injective. This implies that $R / A$ is quasi-Frobenius and so $R$ is uniserial.

\section{References}

[1] K. A. Byrd, 'Some characterizations of uniserial rings', Math. Ann. 186 (1970), 163-170.

[2] C. Faith and E. A. Walker, 'Direct sum representatives of injective modules', J. Algebra 5 (1967), 203-221.

[3] C. Faith, 'Rings with ascending chain condition on annihilators', Nagoya Math. J. 27 (1966), 179-191.

[4] C. Faith, Algebra II. Ring Theory, (Springer-Verlag, New York, 1967).

[5] K. R. Fuller, 'On direct representations of quasi-injectives and quasi-projectives', Arch. Math. 20 (1969), 495-502.

[6] K. R. Fuller, 'On indecomposable injectives over artinian rings', Pacific J. Math. 29 (1969), 115-135.

[7] K. R. Fuller and D. A. Hill, 'On quasi-projective modules via relative projectivity', Arch. Math. 21 (1970), 369-373.

[8] V. K. Goel and S. K. Jain, ' $\pi$-injective modules and rings whose cyclic modules are $\pi$-injective', Comm. Algebra 6 (1978), 59-73.

[9] M. Harada, 'Non-small modules and non-cosmall modules', Ring Theory, pp. 669-689, (Proceedings of 1978 Antwerp conference, Marcel Dekker, 1979).

[10] A. Koehler, 'Quasi-projective and quasi-injective modules', Pacific J. Math. 36 (1971), 713-720.

[11] L. S. Levy, 'Commutative rings whose homomorphic images are self-injective', Pacific J. Math. 18 (1966), 149-153.

[12] S. Mohamed and B. J. Mueller, 'Direct sums of dual-continuous modules', Math. Z. 178 (1981), 225-232.

[13] S. Mohamed, B. J. Mueller and S. Singh, 'Quasi-dual continuous modules', J. Austral. Math. Soc. Ser. A 39 (1985), 287-299. 
[14] S. Mohamed and S. Singh, 'Generalizations of decomposition theorems known over perfect rings', J. Austral. Math. Soc. Ser. A 24 (1977), 496-510.

[15] S. Mohamed and B. J. Mueller, Continuous and discrete modules, London Math. Soc. Lecture Notes Series No. 147, (Cambridge University Press, Cambridge, 1990).

[16] K. Oshiro, 'Lifting modules, extending modules and their applications to generalized uniserial rings', Hokkaido Math. J. 13 (1984), 339-346.

Ohio University

Ohio University

Athens, Ohio 45701 Athens, Ohio 45701

U.S.A.

U.S.A.

Ohio State University

Lima, Ohio 45804

U.S.A. 